\title{
A simplified model of precipitation enhancement over a heterogeneous surface
}

\author{
Guido Cioni ${ }^{1,2,3}$ and Cathy Hohenegger ${ }^{1,3}$ \\ ${ }^{1}$ Max Planck Institute for Meteorology, Hamburg, Germany \\ ${ }^{2}$ International Max-Planck Research School on Earth System Modelling, Hamburg, Germany \\ ${ }^{3}$ Hans-Ertel-Zentrum for Weather Research
}

Correspondence: Guido Cioni (guido.cioni@mpimet.mpg.de)

Received: 6 September 2017 - Discussion started: 11 September 2017

Revised: 12 May 2018 - Accepted: 26 May 2018 - Published: 7 June 2018

\begin{abstract}
Soil moisture heterogeneities influence the onset of convection and subsequent evolution of precipitating systems through the triggering of mesoscale circulations. However, local evaporation also plays a role in determining precipitation amounts. Here we aim at disentangling the effect of advection and evaporation on precipitation over the course of a diurnal cycle by formulating a simple conceptual model. The derivation of the model is inspired by the results of simulations performed with a high-resolution $(250 \mathrm{~m})$ large eddy simulation model over a surface with varying degrees of heterogeneity. A key element of the conceptual model is the representation of precipitation as a weighted sum of advection and evaporation, each weighed by its own efficiency. The model is then used to isolate the main parameters that control precipitation variations over a spatially drier patch. It is found that these changes surprisingly do not depend on soil moisture itself but instead purely on parameters that describe the atmospheric initial state. The likelihood for enhanced precipitation over drier soils is discussed based on these parameters. Additional experiments are used to test the validity of the model.
\end{abstract}

\section{Introduction}

Will a wetter soil lead to more or less precipitation? This apparently simple question inspired many studies over the course of the last 50 years. Over a homogeneous surface, precipitation is expected to increase with surface evaporation, and thus with soil moisture in a soil-moisture-limited regime (Manabe, 1969; Budyko, 1974), regardless of the at- mospheric state (Cioni and Hohenegger, 2017) as long as convection can be triggered on both dry or wet surfaces (Findell and Eltahir, 2003). However, the real world is far from being homogeneous. The presence of heterogeneity in surface soil moisture induces thermally driven mesoscale circulations (Segal and Arritt, 1992) which transport moist air from spatially wetter patches to spatially drier patches, acting against the initial perturbation of soil moisture, and which can then affect the distribution of precipitation.

Many idealized studies have investigated the effect of such circulations on convection and ensuing precipitation. Avissar and Liu (1996) found that the land-surface wetness heterogeneity (i.e., spatial gradients of soil moisture) controls the transition from a randomly scattered state of convection to a more organized one where clouds form ahead of the front associated with the mesoscale circulation. The presence of such circulations also tends to enhance the precipitation amount. Further analyses have shown that this basic response can be modified by many environmental factors.

Yan and Anthes (1988) found that accumulated precipitation is maximized over spatially dry patches when the patch length is comparable to the local Rossby radius of deformation ( $\sim 100 \mathrm{~km}$ in mid-latitudes), a result that was later confirmed by Chen and Avissar (1994) and Lynn et al. (1998). Robinson et al. (2008) proposed an alternative explanation by which the effect of surface hot spots is maximized for wavelengths of roughly $50 \mathrm{~km}$, that is when the aspect ratio of the applied heating matches the ratio of vertical and horizontal wavenumbers demanded by the dispersion relation for buoyancy (gravity) waves. 
Froidevaux et al. (2014) explored the interaction between horizontal soil moisture variations, wind and precipitation. They found that, only when winds are too weak to control the propagation of thunderstorms, more precipitation is observed over drier surfaces. Finally, the response of precipitation also depends upon the background atmospheric profile. Chen and Avissar (1994) found that the presence of a moist atmospheric profile over a spatially drier surface reduces the precipitation advantage as the surface heat fluxes, which drive the surface heating and thus the circulation, are reduced. Hence, from such studies, an increase in precipitation over spatially drier patches is maximized when the gradient of surface wetness is high, the soil moisture heterogeneity length scale is around $50-100 \mathrm{~km}$ and no background wind is present.

These same mechanisms can be observed in some areas of the world, the so-called hot spots of land-atmosphere interactions (Koster et al., 2004). Several observational studies (e.g., Taylor et al., 2012) showed that in the Sahel region, thunderstorms occur preferably over regions drier than their surroundings. In other areas of the world the synoptic forcing is usually so strong that a robust relationship of causality between soil moisture and precipitation cannot be found (Tuttle and Salvucci, 2017). Instead of speaking of heterogeneous or homogeneous conditions, Guillod et al. (2015) have indicated that over most areas of the world, except the Sahel, a negative spatial coupling coexists together with a positive temporal coupling. That is, areas drier than their surroundings (spatial component) but wetter than the climatological value (temporal component) may receive more precipitation than other areas.

Although the aforementioned studies have qualitatively shown how precipitation is influenced by soil moisture, soil moisture gradients and by the atmospheric environment, here we aim at developing a simplified conceptual model to formally isolate the control of soil moisture on precipitation. In particular we aim at developing a mathematical expression for the derivative of precipitation with respect to soil moisture in the case of a heterogeneous surface to understand the response of precipitation to soil moisture changes. In this case, precipitation is not only affected by the advection of moisture due to the mesoscale circulation but also by local evaporation (Wei et al., 2016). These two factors depend differently on soil moisture.

The mesoscale circulation triggered by the surface wetness heterogeneity strengthens with decreasing soil moisture of the dry patch, as this gives a larger spatial gradient of surface heat fluxes and thus of surface pressure. Instead local evaporation is limited with reduced local soil moisture. The superposition of local evaporation and remote moisture advection eventually contribute to the observed precipitation, with the atmosphere being the medium that weighs these two different contributions.
Lintner et al. (2013) already derived an equation for the derivative of precipitation with respect to soil moisture based on a model of intermediate-level complexity of the tropical atmosphere (the Quasi-equilibrium Tropical Circulation Model 1, Neelin and Zeng, 2000). Inspired by their work, we develop a theoretical model which is based on similar assumptions but simplifies the formulation of moisture advection and evaporation. In particular the fact that we consider the specific case of advection by a thermally induced mesoscale circulation, and not by the large-scale flow, will allow us to greatly simplify the idealized framework.

Section 2 describes the model and experimental setup that allows us to simulate the evolution of convective clouds and precipitation over a heterogeneous land surface during a diurnal period. After a brief analysis of the features of the convective diurnal cycle in Sect. 3.1 we estimate the various terms of the moisture balance and in particular the efficiencies of the conversion of evaporation and advection into precipitation in Sect. 3.2. These results are used in Sect. 4 to derive a simple conceptual model of how precipitation responds to soil moisture changes over a heterogeneous surface. We will show that, at least to a first order, the change of precipitation with soil moisture does not depend on the soil moisture content itself but only on the atmospheric state. The results are concluded in Sect. 5.

\section{Methods}

The modeling framework used in this work is, in terms of physical parametrizations and dynamical core, identical to the one described in Cioni and Hohenegger (2017), to which the reader is referred for details. We use ICON-LEM (Heinze et al., 2017) as atmospheric model coupled to the land-surface model, TERRA-ML, to simulate the diurnal cycle of convection over idealized land surfaces from 06:00 to 24:00 LST (Local Solar Time).

The horizontal periodic domain spans $1600 \times 400$ points with a resolution (in terms of the triangle edges; see Zängl et al., 2015) of $250 \mathrm{~m}$, which results in a size of approximately $400 \mathrm{~km} \times 100 \mathrm{~km}$. In the vertical dimension $150 \mathrm{lev}-$ els are distributed from the surface up to the model top, located at $21 \mathrm{~km}$ : the spacing reaches $20 \mathrm{~m}$ in the lower levels and $400 \mathrm{~m}$ close to the model top. In contrast to Cioni and Hohenegger (2017), heterogeneous surface conditions are used as bottom boundary conditions. The heterogeneity is prescribed by dividing the domain's $x$ direction into two patches having the same surface area of $200 \times 100 \mathrm{~km}^{2}$. Figure 1 displays a sketch of the domain setup, together with a visual representation of convective features that will be discussed later.

The domain is rectangular in order to limit computational expenses and is elongated in the $x$ axis given that the front associated with the simulated mesoscale circulation is expected to propagate with a direction parallel to the $x$ axis. The cho- 


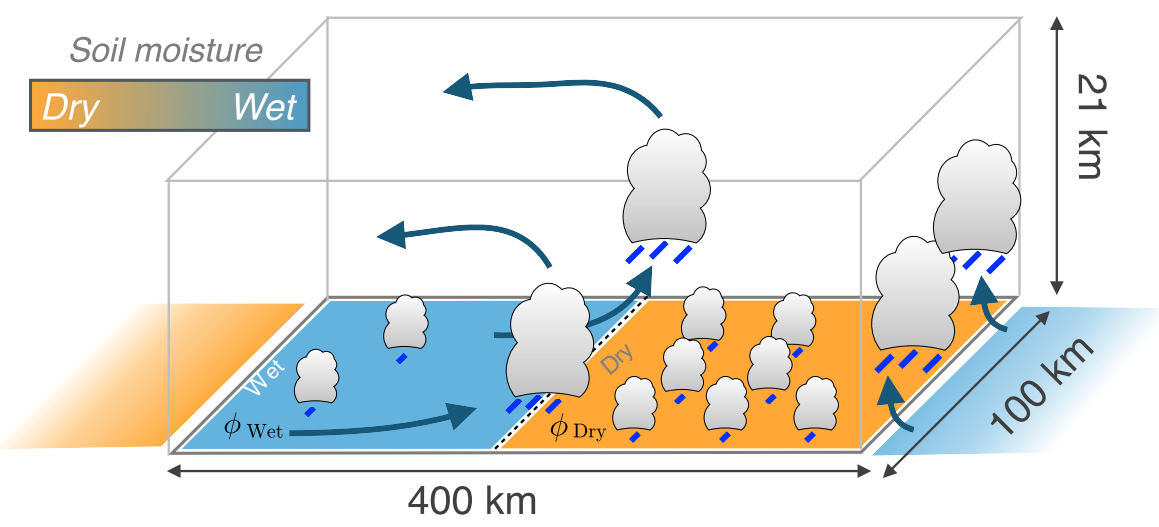

Figure 1. Idealized sketch of the employed experimental framework. The initial condition for soil moisture and the expected initial development of convection are also sketched in order to ease the interpretation of the results.

sen patch size of $200 \mathrm{~km}$ is larger than the optimal value of the heterogeneity wavelength $(\sim 100 \mathrm{~km})$ identified by Chen and Avissar (1994), Yan and Anthes (1988) and Lynn et al. (1998). Therefore, we do not expect to maximize, in terms of the strength of the mesoscale circulation, the response of the atmosphere to the surface heterogeneity. This could eventually reduce the dynamic contribution of advection on precipitation. The larger domain has nevertheless the advantage that the opposite fronts collide later in the day so that the daily precipitation amounts are less affected by what happens after the fronts have collided. The sensitivity of the diurnal evolution of precipitation to different $y$-axis size was tested, and found to not affect the results.

The surface heterogeneity is introduced by setting two different initial values of volumetric soil moisture $\phi\left(\mathrm{m}^{3} \mathrm{~m}^{-3}\right)$ for the two patches, $\phi_{\text {wet }}$ and $\phi_{\text {dry }}$, respectively. The value is set to the entire soil column to ease the interpretation of the results. The other parameters that characterize the land surface, including for example soil temperature, are horizontally homogeneous over the entire domain. Initially the soil temperature is prescribed using a linear profile which includes a climatological layer with a temperature of $281 \mathrm{~K}$ at $14.58 \mathrm{~m}$ below the surface and a surface layer which has the same temperature as the overlying lowermost level of the atmosphere (see Cioni and Hohenegger, 2017).

The atmospheric initial state is spatially homogeneous except for random perturbations added to the vertical velocity and the virtual potential temperature in the lowermost three levels to break the perfectly symmetric initial state. The atmosphere is initialized using the dry soil advantage profile of Findell and Eltahir (2003), albeit with winds set to zero to simplify the analysis (see Fig. 2). This sounding, indicated throughout the manuscript as DA, was observed on 23 July 1999 in Lincoln (Illinois, USA) and was chosen as a typical example by Findell and Eltahir (2003) for cases when a strong heating of a homogeneous surface favors the triggering of convection.
To study the response of precipitation to variations in soil moisture, we perform a set of experiments by setting $\phi_{\text {wet }}$ to the saturation value at the initial time and varying $\phi_{\text {dry }}$, with values ranging from the saturation to $20 \%$ of the saturation value. The latter value is below the wilting point for the chosen soil type (loam). More details about the soil type can be found in Cioni and Hohenegger (2017) and Doms et al. (2011). The upper part of Table 1 summarizes the simulations performed with this basic configuration.

In order to test the validity of the theory proposed in Sect. 4, based on this set of basic experiments, we perform further sensitivity experiments. First, we decrease the initial value of $\phi_{\text {wet }}$ to $70 \%$ of the saturation value. Second, we change the initial atmospheric profile. We tested the wet soil advantage sounding of Findell and Eltahir (2003) where, in contrast to the dry soil advantage sounding, convection triggering requires a strong moistening of the boundary layer. We also tested the sounding of Schlemmer et al. (2012), indicated as ID, which represents an idealization of the typical atmospheric state prone to convection in Europe (see Fig. 2b). This sounding thus greatly differs from the conditions as observed in Lincoln. It has a lower surface temperature and a lower integrated water vapor content but a larger initial instability. As the use of the wet soil advantage sounding of Findell and Eltahir (2003) yields very similar results as in DA, which is not the case when using ID, we only report here on the ID simulations.

\section{Results}

\subsection{General features of convection}

Here we describe the general features of the extreme case, DA_20_100, which reproduces the features expected from these kinds of simulations. The differential heating of the two patches, caused by the heterogeneity in soil moisture, manifests itself as a gradient of both sensible and latent heat 

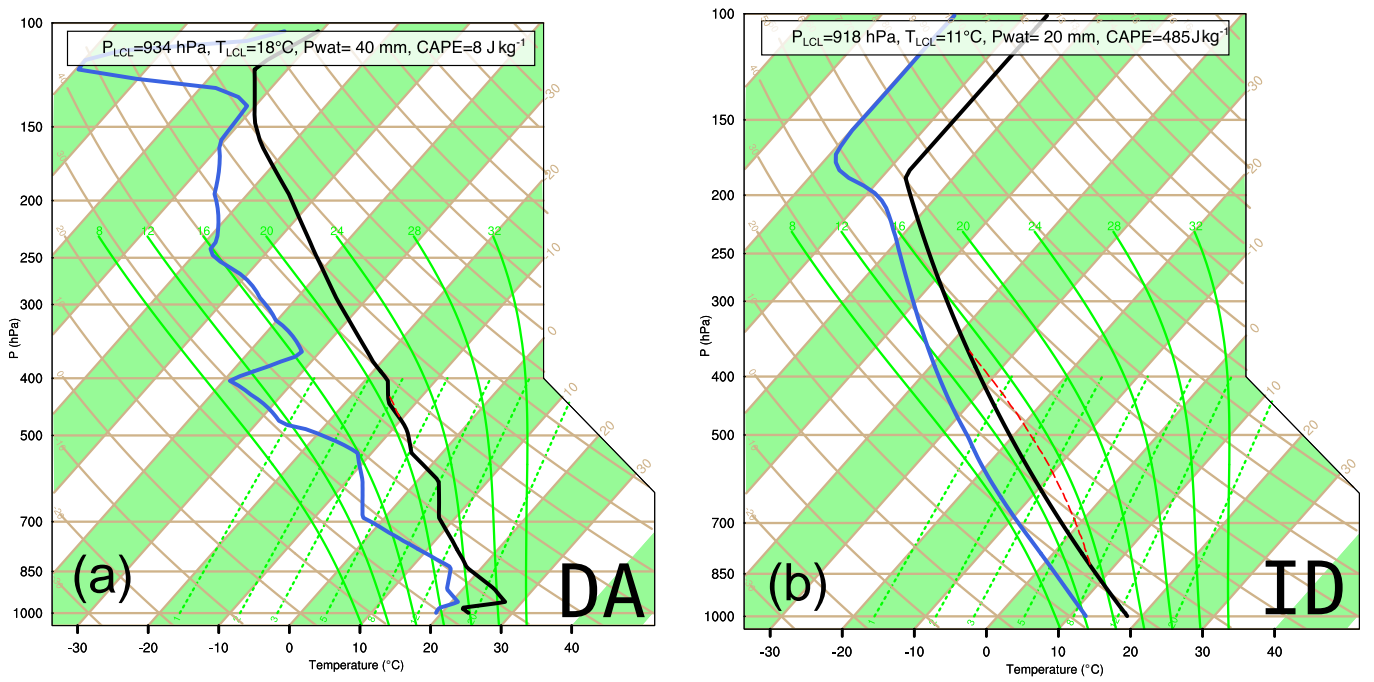

Figure 2. Skew-T diagrams of the two soundings used to initialize the atmosphere in the simulations. (a) Shows the dry soil advantage sounding of Findell and Eltahir (2003), DA, while (b) shows the idealized sounding of Schlemmer et al. (2012), ID. The upper inset in both panels show the value of pressure at the LCL (lifting condensation level), temperature at the LCL, precipitable water and CAPE (convective available potential energy).

fluxes. At 12:00 LST the difference in sensible heat flux between the two patches reaches almost $280 \mathrm{~W} \mathrm{~m}^{-2}$. This results in a difference in near-surface virtual potential temperature of about $4 \mathrm{~K}$ at the same time (see the colored contours in Fig. 3). As a consequence, a pressure gradient of about $1 \mathrm{hPa}$ develops close to the surface, which supports a thermally driven circulation (Segal and Arritt, 1992). The circulation consists of a front of moist air moving inland over the dry patch at lower levels (from the surface up to $1 \mathrm{~km}$ ) and a return flow between 1 and $3 \mathrm{~km}$, as shown by the wind vectors in Fig. 3. As a result of the circulation, and as found in past studies, convection preferentially develops over the dry patch and in particular at the edge of the front associated with the mesoscale circulation.

In order to track the front associated with the mesoscale circulation we use an algorithm designed to follow one of the fronts moving over the dry patch. The algorithm is based on the $y$-averaged zonal wind speed at $150 \mathrm{~m}$ of height. It is triggered when the wind speed in the middle of the domain reaches $1 \mathrm{~m} \mathrm{~s}^{-1}$ and automatically stops when the opposite fronts collide in the center of the dry patch. At every output time step (15 $\mathrm{min})$ a search of the maximum value of zonal wind speed is performed in a box which is suitably chosen in order to maintain the focus of the tracking algorithm on the front.

More specifically, at the first two time instants the maximum is searched over the entire dry patch, while from the third time step onward the maximum search is performed in a box centered on a first guess obtained from a simple linear extrapolation of the previous time instants. The size of the box is the only parameter that needs to be tuned when tracking the front in different simulations. Otherwise, the al-

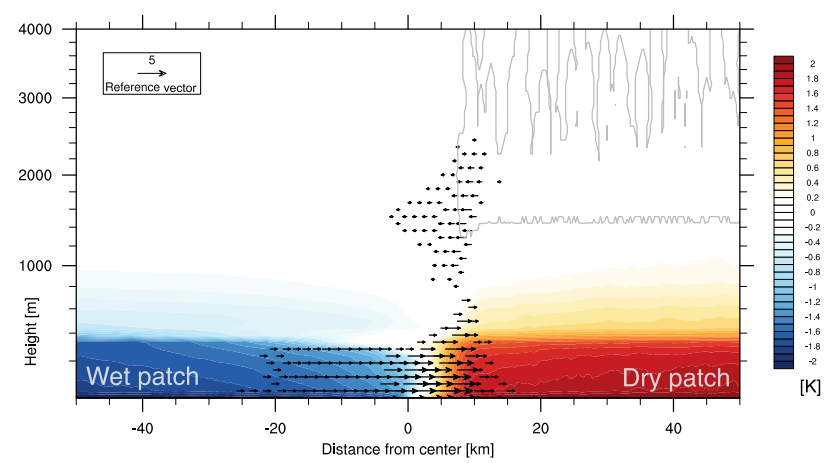

Figure 3. $x-z$ diagram at 12:00 LST of $y$-averaged quantities for the DA_20_100 case. Zonal temperature anomaly (color contours), zonal wind (vectors, values between -0.5 and $1 \mathrm{~m} \mathrm{~s}^{-1}$ are masked) and cloud water mixing ratio (grey contour, only $10^{-5} \mathrm{~g} \mathrm{~kg}^{-1}$ isoline). On the $x$-axis, numbers indicate the distance from the center of the domain in $\mathrm{km}$.

gorithm is robust. As an example, in the DA_20_100 case shown in Fig. 4, the box comprises five grid points, thus approximately $1.25 \mathrm{~km}$.

Figure 4a shows the Hovmöller diagram of the zonal wind and the tracked position of the front every $15 \mathrm{~min}$ with shaded circles for the case DA_20_100. In Fig. 4b the position and speed of the front obtained with the aforementioned algorithm are displayed. The front starts to slowly propagate in the late morning with a velocity smaller than $2 \mathrm{~m} \mathrm{~s}^{-1}$ but is later accelerated by cold pools, in agreement with Rieck et al. (2015). The cold pools are formed after the first strong precipitation event between 12:00 and 13:00 LST. The speed of 
Table 1. Overview of the performed simulations. The first column indicates the experiment name, whereas the second column indicates the sounding used for initialization: DA for dry soil advantage, after Findell and Eltahir (2003), and ID for idealized, after Schlemmer et al. (2012). Third and fourth columns indicate the value of soil moisture over the dry and wet patches, respectively, in percentage of the saturation value. The naming convention for the experiments follows SOUNDING_ $\phi_{\text {dry_}} \phi_{\text {wet }}$. The vertical lines that characterize the ID cases are used to omit the repetition of the same experiments description, i.e., ID_30_100, ID_40_100, etc.

\begin{tabular}{llrr}
\hline Experiment & Sounding & $\phi_{\text {dry }}$ & $\phi_{\text {wet }}$ \\
\hline \multicolumn{2}{l}{ Basic configuration } & & \\
\hline DA_20_100 DA & 20 & 100 \\
DA_30_100 & & 30 & 100 \\
DA_40_100 & 40 & 100 \\
DA_50_100 & 50 & 100 \\
DA_60_100 & 60 & 100 \\
DA_65_100 & 65 & 100 \\
DA_70_100 & 70 & 100 \\
DA_80_100 & 80 & 100 \\
DA_100_100 & & 100 & 100 \\
\hline Unsaturated wet patch & & \\
\hline DA_20_70 & DA & 20 & 70 \\
DA_30_70 & & 30 & 70 \\
DA_40_70 & & 40 & 70 \\
DA_50_70 & & 50 & 70 \\
DA_60_70 & & 60 & 70 \\
DA_70_70 & & 70 & 70 \\
\hline Idealized sounding & & \\
\hline ID_20_100 & ID & 20 & 100 \\
I & I & 100 & 100 \\
ID_100_100 & ID & & \\
\hline
\end{tabular}

the front reaches values of up to $7 \mathrm{~m} \mathrm{~s}^{-1}$ before the front collides with the opposing front coming from the outer boundary due to the periodic domain. When the soil moisture of the dry patch exceeds $70 \%$ of the saturation value no circulation forms because the gradient in surface temperature is too weak to cause a pressure difference between the patches. In this case the convection transitions to a randomly scattered state (Avissar and Liu, 1996) and we define the speed of the front to be $0 \mathrm{~m} \mathrm{~s}^{-1}$.

\subsection{Local and remote sources of precipitation}

The diurnal cycle of precipitation can be inspected and compared to the one of evaporation and advection, using the methodology introduced in Appendix A. This is needed to later formally express precipitation as a function of soil moisture (see Sect. 4). Figure 5 shows the various components of the moisture balance computed every $5 \mathrm{~min}$ from the model output and averaged over the dry patch as well as over the
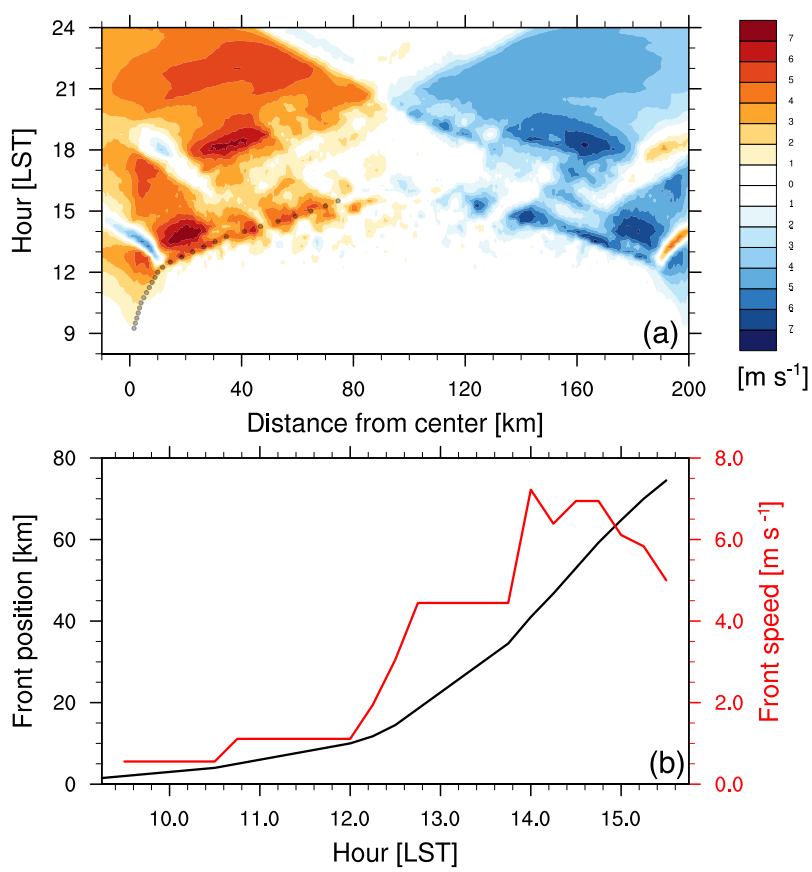

Figure 4. Tracking of the front associated with the mesoscale circulation for the case DA_20_100. (a) Hovmöller diagram (distance from domain center vs. time) of the $y$-averaged zonal wind at a height of $150 \mathrm{~m}$ above the surface. Dots indicate the position of the front tracked every $15 \mathrm{~min}$ (see text for details). (b) Front inland propagation (black line) with respect to the center of the domain $(\mathrm{km})$ and front speed (red line) derived using finite differences $\left(\mathrm{m} \mathrm{s}^{-1}\right)$.

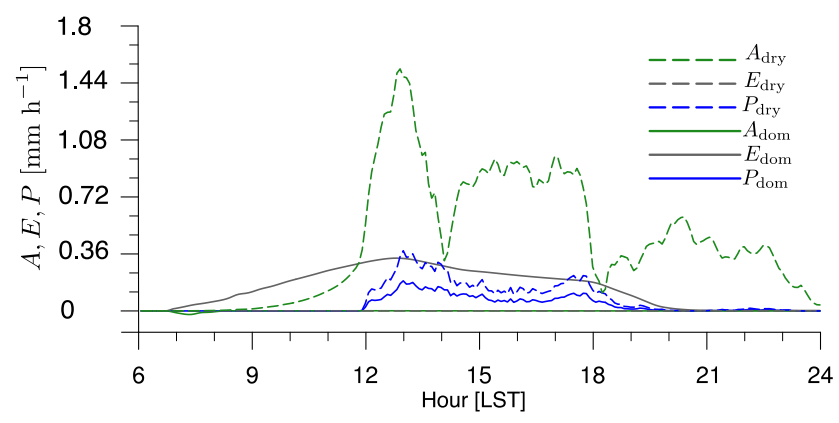

Figure 5. Different terms of the moisture balance (Eq. A3) computed for the entire domain (subscript dom, solid lines) and for the dry patch (subscript dry, dashed lines) in the DA_20_100 case. $A$ indicates advection, $E$ evaporation and $P$ precipitation. Units are millimeters per hour. Note that all variables in this figure are instantaneous.

entire domain. It can be verified that the advection term averaged over the entire domain is zero, as expected. Instead, when considering the residual averaged over the dry patch, $A_{\text {dry }}$, it is always positive, indicating a net transport of moisture from the wet to the dry patch.

The advection of moisture over the dry patch increases in the late morning as a result of the propagation of the front 


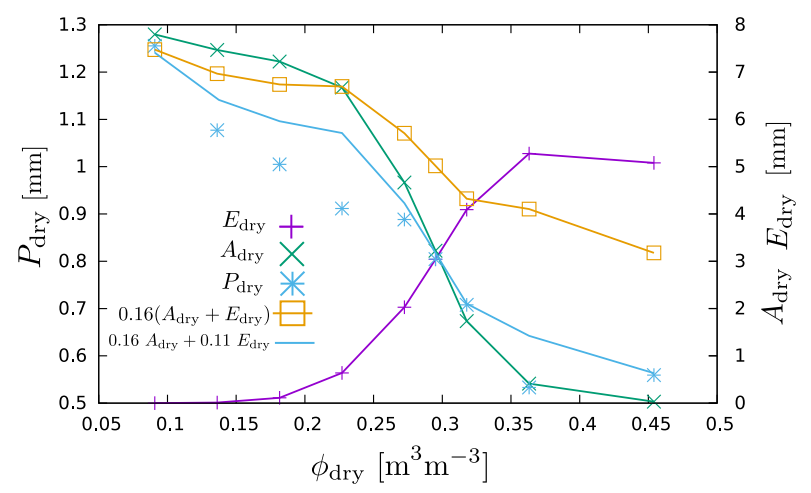

Figure 6. Values of advection (green line and crosses), evaporation (purple line and plus symbols) and precipitation (blue asterisks) from Table 2 as a function of soil moisture. The orange line represents an estimate of precipitation obtained as a sum of advection and evaporation weighted by the same efficiency, i.e., $0.16\left(A_{\mathrm{dry}}+E_{\mathrm{dry}}\right)$, while the blue line represents a similar estimate obtained by using two different efficiencies, i.e., $0.16 A_{\text {dry }}+0.11 E_{\text {dry }}$.

(see Fig. 4) and reaches a maximum at around 13:00 LST. This behavior is similar to the one observed by Yan and Anthes (1988, their Fig. 9). The first deep convection event in DA_20_100 between 12:00 and 13:00 LST produces a strong cold pool which causes a strong surface divergence, explaining the minimum at about 14:00 LST in Fig. 5. Given that the maximum of precipitation associated with this event is located in the vicinity of the boundary between the wet and the dry patch, this induces a net negative effect on $A_{\text {dry }}$.

In order to study the variation in the moisture budget terms as a function of $\phi_{\text {dry }}$ we conduct the same moisture balance analysis for every simulation and integrate the values over the entire diurnal cycle (18 h). Results are reported in Table 2. As expected the advection term decreases with increasing local soil moisture, whereas local evaporation increases. Overall the accumulated precipitation averaged over the dry patch decreases when the soil moisture increases, as shown also in Fig. 6. The sharp decrease in precipitation with increasing values of soil moisture seems to suggest that advection and evaporation are characterized by different weights when producing precipitation. In fact, if the contribution of these processes would be the same, we would expect to observe a flattening of the precipitation values (blue asterisks in Fig. 6) instead of a decrease. In other words, advection appears to be more efficient than evaporation in producing precipitation, as the increase in $E_{\text {dry }}$ with soil moisture is followed by a sharp decrease in $P_{\text {dry }}$.

These qualitative observations can be formalized by defining the precipitation efficiency. This approach was first proposed by Budyko (1974) and later adopted by many studies including the one of Schär et al. (1999). The overall assumption underlying the pioneering work of Budyko (1974) is that
Table 2. Values of soil moisture $\left(\mathrm{m}^{3} \mathrm{~m}^{-3}\right)$, advection ( $\left.\mathrm{mm}\right)$, evaporation $(\mathrm{mm})$ and precipitation $(\mathrm{mm})$ over the dry patch accumulated over the diurnal cycle. The rightmost column shows the precipitation efficiency (dimensionless) computed as $\frac{P_{\mathrm{dry}}}{A_{\mathrm{dry}}+E_{\mathrm{dry}}}$.

\begin{tabular}{lcrccc}
\hline Case & $\phi_{\text {dry }}$ & $A_{\text {dry }}$ & $E_{\text {dry }}$ & $P_{\text {dry }}$ & $\eta$ \\
\hline DA_20_100 & 0.0908 & 7.796 & 0.0008 & 1.255 & 0.161 \\
DA_30_100 & 0.1362 & 7.467 & 0.0113 & 1.077 & 0.144 \\
DA_40_100 & 0.1816 & 7.223 & 0.1140 & 1.005 & 0.137 \\
DA_50_100 & 0.2270 & 6.673 & 0.6373 & 0.912 & 0.125 \\
DA_60_100 & 0.2724 & 4.665 & 2.0271 & 0.888 & 0.133 \\
DA_65_100 & 0.2951 & 3.222 & 3.0393 & 0.805 & 0.129 \\
DA_70_100 & 0.3178 & 1.734 & 4.0920 & 0.708 & 0.122 \\
DA_80_100 & 0.3632 & -0.412 & 5.2770 & 0.533 & 0.094 \\
DA_100_100 & 0.4540 & 0.031 & 5.0800 & 0.560 & 0.110 \\
\hline
\end{tabular}

moisture coming from inside (local evaporation) or outside (remote advection) of some closed domain is well mixed. Under this assumption one can express the precipitation over a certain area as

$P_{\text {area }}=\eta\left(A_{\text {area }}+E_{\text {area }}\right)$,

where $\eta$ is the precipitation efficiency. All the terms are considered as areal averages and integrated over a certain time period. The rightmost column of Table 2 shows the efficiency $\eta$ computed according to Eq. (1). It can be seen that, in this case, convection is not so efficient in converting local and remote sources of moisture into precipitation as the values range from 16 to $9 \%$. More importantly, the efficiency values vary by up to $7 \%$ depending on the initial $\phi_{\text {dry }}$. In fact, in the case DA_20_100, evaporation over the dry patch is negligible, i.e., $E_{\mathrm{dry}} \simeq 0$, so that Eq. (1) applied to the dry patch reads $P_{\text {dry }} \simeq \eta A_{\text {dry }}$. Thus, the efficiency obtained in this case is representative of the advection process and can be interpreted as an advection efficiency $\eta_{\mathrm{A}}$. On the other hand, in DA_100_100 the advection is negligible so that in this case we obtain an evaporation efficiency $\eta_{\mathrm{E}}$. Taking all these findings together, we rewrite Eq. (1) as

$P_{\text {area }}=\eta_{\mathrm{A}} \cdot A_{\text {area }}+\eta_{\mathrm{E}} \cdot E_{\text {area }}$,

where now $\eta_{\mathrm{A}} \neq \eta_{\mathrm{E}}$. The values estimated from Table 2 are $\eta_{\mathrm{A}}=0.16$ and $\eta_{\mathrm{E}}=0.11$.

Figure 6 confirms that, regardless of the particular choice of a single efficiency $\eta$, the decrease in precipitation over wetter soils cannot be captured (orange line in Fig. 6). In contrast, using the two efficiencies, $\eta_{\mathrm{A}}$ and $\eta_{\mathrm{E}}$, gives a much better match with the simulated value of $P_{\text {dry }}$ (see blue line in Fig. 6). Also, by using two efficiencies, the latter become independent of soil moisture. The efficiencies can be alternatively estimated through a fit of Eq. (2) using all the values of $A_{\text {dry }}$ and $E_{\text {dry }}$ in Table 2 . In this case we obtain the values $\eta_{\mathrm{A}}=0.15$ and $\eta_{\mathrm{E}}=0.10$ which, as expected, do not differ much from the ones computed using the two extreme cases. 
Table 3. As in Table 2 but for the ID sounding.

\begin{tabular}{lrrrc}
\hline Case & $A_{\text {dry }}$ & $E_{\text {dry }}$ & $P_{\text {dry }}$ & $\eta$ \\
\hline ID_20_100 & 3.814 & 0.008 & 1.789 & 0.468 \\
ID_30_100 & 3.861 & 0.028 & 1.912 & 0.492 \\
ID_40_100 & 3.920 & 0.143 & 1.671 & 0.411 \\
ID_50_100 & 3.557 & 0.659 & 1.740 & 0.413 \\
ID_60_100 & 2.631 & 2.054 & 1.759 & 0.376 \\
ID_70_100 & 0.865 & 4.080 & 1.542 & 0.312 \\
ID_80_100 & -0.068 & 4.884 & 1.652 & 0.334 \\
ID_100_100 & 0.022 & 4.776 & 1.662 & 0.346 \\
\hline
\end{tabular}

The fact that one efficiency is not enough to describe the variations in precipitation, in contrast to previous studies, may be linked to the fact that we consider a small domain and a short timescale. The assumption of a well-mixed atmosphere likely holds better on a continental (e.g., Europe) and seasonal scales, as in Schär et al. (1999). Using two efficiencies nevertheless requires data from at least two simulations with different values of advection, evaporation and precipitation.

Initializing the atmosphere with a different sounding will likely lead to different efficiencies. This is illustrated with the ID_cases (see Table 3), where the idealized sounding of Schlemmer et al. (2012) is used to initialize the atmosphere (see Sect. 2). For a given soil moisture, advection reaches smaller values that in the DA case. This is mainly an effect of larger precipitation amounts that fall on the wet patch which in turn prevents an efficient advection of moisture from the wet to the dry patch.

The efficiencies computed for this case range from 47 to $31 \%$, indicating that the atmosphere is more efficient at converting advection and evaporation into precipitation than in $D A$. The higher efficiencies obtained with the ID sounding are due to a combination of different effects. One of those is the different convection triggering. With the ID sounding convection is triggered almost $1 \mathrm{~h}$ before compared to the DA sounding (not shown). This allows the atmosphere to fully exploit the instability caused by the morning heating which manifests itself as a stronger enhancement of precipitation at the front, as shown in Fig. 7. This is also corroborated by the fact that convective available potential energy (CAPE) at 15:00 LST is larger than the one at the initial time over both patches in DA_20_100, whereas it is depleted over the dry patch in the ID_20_100 case (not shown).

Moreover, as indicated by Fig. 2, the dew-point depression in the ID sounding is smaller than in the DA sounding throughout most of the atmospheric column. This suggests that, in the ID case, convective updrafts are less affected by the entrainment of environmental dry air. We verify this by computing the average difference in MSE (moist static energy) between updrafts, defined as grid points with vertical velocities greater than $1 \mathrm{~m} \mathrm{~s}^{-1}$ and cloud water content

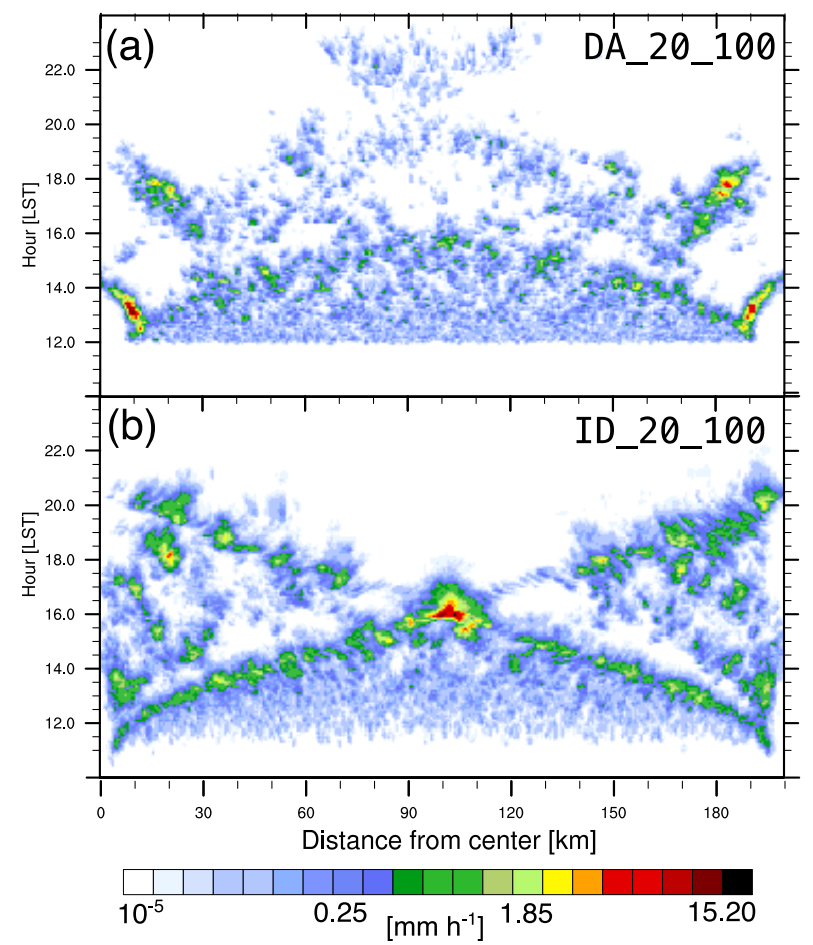

Figure 7. Hovmöller diagram of precipitation rate $\left(\mathrm{mm} \mathrm{h}^{-1}\right)$ in cases (a) DA_20_100 and (b) ID_20_100.

greater than $10^{-4} \mathrm{~kg} \mathrm{~kg}^{-1}$, and the environment (not shown). Results show that this difference in the ID case is less than $50 \%$ of the values observed in the DA case. Our goal, however, is not to determine how the efficiencies depend on the atmospheric state but rather how precipitation depends on the efficiencies.

Despite the differences between DA and ID, the ID case confirms that advection and evaporation exhibit distinct efficiencies and that precipitation decreases with increased local soil moisture. Here the decrease in precipitation is smaller than the one obtained in the DA_ cases. Although this could be related to a weaker sensitivity of the ID atmospheric state to modifications in the land-surface heterogeneity, we note that the amount of precipitation strongly depends on the collision of the fronts. As shown in Fig. 7 the collision of the fronts in the center of the dry patch has different effects on precipitation depending on the atmospheric state. In the ID_20_100 case strong precipitation events with local maxima of $10 \mathrm{~mm} \mathrm{~h}^{-1}$ are produced in the center of the patch after the fronts' collision and several secondary events develop due to the fronts propagating away from the collision. Instead, in the DA_20_100 case, no strong precipitation event is produced when the fronts collide. 


\section{Conceptual model}

In Sect. 3.2 we showed that precipitation can be expressed as a linear combination of advection and evaporation weighted by different efficiencies which are assumed independent of soil moisture (Eq. 2). Knowing this we can now try to answer the first question that was posed in the Introduction: what are the minimum parameters that control the variation in precipitation with soil moisture? In order to do so we first have to derive some functional forms of evaporation and advection in terms of soil moisture.

\subsection{Surface evaporation}

The simplest parametrization of evaporation (we will neglect the transpiration part given that our study does not include plants) is the so-called bucket model introduced by Budyko (1961) and extended by Manabe (1969). Evaporation is defined as a potential term controlled by a limiting factor (also called stress factor). Here we use such a formulation to first approximate the surface latent heat flux $\mathrm{LH}\left(\mathrm{mm} \mathrm{h}^{-1}\right)$ at a certain point in space and time as a function of soil moisture $\phi\left(\mathrm{m}^{3} \mathrm{~m}^{-3}\right)$ :

$\mathrm{LH}(\phi)=\mathcal{A} Q_{\text {net }} \times \begin{cases}0 & \text { for } \phi<\phi_{\mathrm{wp}} \\ \frac{\phi-\phi_{\mathrm{wp}}}{\phi_{\text {crit }}-\phi_{\mathrm{wp}}} & \text { for } \phi_{\mathrm{wp}} \leq \phi \leq \phi_{\text {crit }}, \\ 1 & \text { for } \phi>\phi_{\text {crit }}\end{cases}$

where $Q_{\text {net }}\left(\mathrm{mm} \mathrm{h}^{-1}\right)$ is the net incoming radiation at the surface (longwave + shortwave), $\phi_{\mathrm{wp}}\left(\mathrm{m}^{3} \mathrm{~m}^{-3}\right)$ the soil moisture at the permanent wilting point and $\phi_{\text {crit }}\left(\mathrm{m}^{3} \mathrm{~m}^{-3}\right)$ is the critical soil moisture at which evaporation does not increase any more with increasing soil moisture. As explained by Seneviratne et al. (2010) this does not usually correspond to the field capacity.

$\mathcal{A}$ is a proportionality constant which needs to be introduced and specified given that, even in the extreme case of a saturated soil, non-zero sensible heat fluxes and ground heat flux prevent the entire conversion of $Q_{\text {net }}$ into LH. The constant $\mathcal{A}$ clearly depends on the particular soil model employed as well as on the different parameters that characterize the soil type considered (e.g., albedo, heat capacity) and partially also on the atmosphere.

In order to link Eq. (3) to the accumulated evaporation $E_{\text {dry }}$ needed in Eq. (2) we average Eq. (3) over the dry patch and integrate it over the accumulation period $\tau$. By doing so we assume a constant value for soil moisture and replace it with the value at the initialization time. Such an assumption is motivated by the fact that changes in soil moisture over one diurnal cycle are not expected to be able to significantly feed back on evaporation and precipitation on such a short timescale. The assumption is also well justified as the daily average value of soil moisture remains similar to its initial value (not shown). This gives

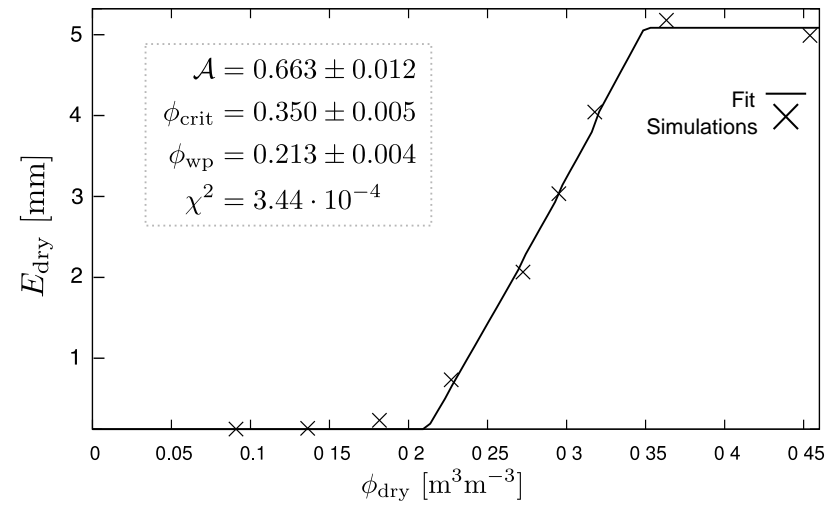

Figure 8. Fit of $E_{\text {dry }}$ with values obtained from the simulations of the default configuration (DA_20_100 to DA_100_100). Crosses indicate values obtained from simulations while the line indicates the fit performed using Eq. (4). The upper left inset shows the values obtained by the fit together with absolute errors and the residual sum of squares $\chi^{2}$, i.e., the sum of the squared difference between the values predicted by the fit and the ones obtained in the simulations.

$E_{\text {dry }}\left(\phi_{\text {dry }}\right)=\tau \mathcal{A}\left\langle Q_{\text {net }}\right\rangle \times \begin{cases}0 & \text { for } \phi_{\text {dry }}<\phi_{\text {wp }} \\ \phi_{\text {dry }}-\phi_{\text {wp }} & \text { for } \phi_{\mathrm{wp}} \leq \phi_{\text {dry }} \leq \phi_{\text {crit }}, \\ \phi_{\text {crit }}-\phi_{\text {wp }} & \text { for } \phi_{\text {dry }}>\phi_{\text {crit }}\end{cases}$

where now $E_{\text {dry }}$ does not depend on time nor space. $\left\langle Q_{\text {net }}\right\rangle$ denotes the net surface incoming radiation averaged over the dry patch and over the period $\tau$, whereas $\phi_{\text {dry }}$ corresponds to the initial value of soil moisture.

Equation (4) can now be used to fit the values of $E_{\text {dry }}$ computed from the simulations (Table 2) to obtain an unambiguous value for the parameters $\mathcal{A}, \phi_{\mathrm{wp}}$ and $\phi_{\text {crit }}$ (see Fig. 8). These are estimated to be $\mathcal{A}=0.663, \phi_{\mathrm{wp}}=0.213 \mathrm{~m}^{3} \mathrm{~m}^{-3}$ and $\phi_{\text {crit }}=0.350 \mathrm{~m}^{3} \mathrm{~m}^{-3}$. Note that the latter estimate is not far from the field capacity of this soil type, i.e., $0.340 \mathrm{~m}^{3} \mathrm{~m}^{-3}$, while the estimated wilting point is almost double the expected one, i.e., $0.110 \mathrm{~m}^{3} \mathrm{~m}^{-3}$. This is related to the fact that the employed bare soil evaporation scheme tends to shut down evaporation too early as noted by Schulz et al. (2016) and Cioni and Hohenegger (2017). Thus, both $\phi_{\mathrm{wp}, \text { crit }}$ depend not only on the employed soil type but also on the soil model.

Figure 8 shows the fit of Eq. (4), together with the values obtained in the simulations. It reveals an excellent agreement between theory and simulations. The small discrepancies mainly come from the fact that we assume a constant value of $\left\langle Q_{\text {net }}\right\rangle=300 \mathrm{~W} \mathrm{~m}^{-2}=0.43 \mathrm{~mm} \mathrm{~h}^{-1}$ across the simulations, although the simulated value depends on soil moisture and varies by about $7 \%$. This is due to different cloud regimes which alter the surface radiation balance (Cioni and Hohenegger, 2017, Sect. 4b).

We note that our formulation of evaporation differs from the one used in the model of Lintner et al. (2013) where potential evaporation was used in place of $Q_{\text {net }}$, which is the 
main difference between the original framework of Budyko (1961) and the one of Manabe (1969).

\subsection{Advection}

Our goal is to find a formulation of $A_{\text {dry }}$ as a function of soil moisture. This can be achieved starting from the definition of Eq. (A2) and assuming that the advection of every tracer is mainly due to the propagation of the front associated with the mesoscale circulation, hence $H=H_{\text {front }}$. In this case

$$
\begin{aligned}
A_{\text {dry }} & =-\left.\frac{1}{\rho_{\mathrm{w}}} \int_{0}^{\tau} \int_{0}^{H_{\text {front }}} \boldsymbol{v}_{\text {front }} \cdot \nabla q_{\text {tot }}\right|_{\text {dry }} \rho_{\mathrm{a}} \mathrm{d} z \mathrm{~d} t, \\
& \simeq-\left.\frac{1}{\rho_{\mathrm{w}}} \int_{0}^{\tau} \int_{0}^{H_{\text {front }}} u_{\text {front }} \frac{\partial q_{\mathrm{tot}}}{\partial x}\right|_{\text {dry }} \rho_{\mathrm{a}} \cdot \mathrm{d} z \mathrm{~d} t,
\end{aligned}
$$

where $H_{\text {front }}$ is the height of the front associated with the mesoscale circulation or, equally, the PBL (planetary boundary layer) height, $\boldsymbol{v}_{\text {front }}$ its speed, $\rho_{\mathrm{a}}$ is the air density and $\rho_{\mathrm{w}}$ the water density. Equation (5) has been already approximated given that the front propagates mainly in the $x$ direction (see Sect. 3.1), so that there is no $y$ component of $\nabla q_{\text {tot }}$.

The propagation speed of the front $u_{\text {front }}$ and $\rho_{\mathrm{a}}$ can be seen as constants in the vertical within the height $H_{\text {front }}$, while $q_{\mathrm{tot}}$ remains a function of $x, z$ and time $t$. The time integration can be replaced by considering the average over time multiplied by the timescale $\tau$ to obtain

$$
\begin{aligned}
A_{\text {dry }} & =-\tau \frac{\rho_{\mathrm{a}}}{\rho_{\mathrm{w}}}\left\langle u_{\text {front }}\right\rangle_{\mathrm{dry}}\left\langle\left.\int_{0}^{H_{\text {front }}} \frac{\partial q_{\text {tot }}}{\partial x}\right|_{\mathrm{dry}} \mathrm{d} z\right\rangle, \\
& =-\tau \frac{\rho_{\mathrm{a}}}{\rho_{\mathrm{w}}}\left\langle u_{\text {front }}\right\rangle_{\mathrm{dry}}\left\langle\int_{0}^{H_{\text {front }}} \frac{\Delta q_{\text {tot }}}{L_{\text {front }}} \mathrm{d} z,\right. \\
& =-\tau \frac{\rho_{\mathrm{a}}}{\rho_{\mathrm{w}}}\left\langle u_{\text {front }}\right\rangle_{\text {dry }} \frac{H_{\text {front }}}{L_{\text {front }}}\left\langle\Delta q_{\text {tot }}\right\rangle .
\end{aligned}
$$

In Eq. (6) we approximated the derivative of $q_{\text {tot }}$ as the difference between the two patches $\Delta q_{\text {tot }}$ divided by the penetration length of the front, $L_{\text {front }}$ (Crosman and Horel, 2010).

To simplify the problem we assume $\Delta q_{\text {tot }} \simeq \Delta q_{\mathrm{v}}$, which is viewed as the difference in specific humidity ahead of the front and behind it. As in studies which have viewed sea breezes as gravity current (Robinson et al., 2013), we assume that this difference is not directly affected by the circulation, which yields an upper bound estimate given that the propagation of the front over the dry patch will act to reduce the gradient in specific humidity in the PBL. The changes in $q_{\mathrm{v}}$ due to surface evaporation accumulated up to a certain time $\tau$ can then be written as

$$
\begin{aligned}
q_{\mathrm{v}}(\tau) & =q_{\mathrm{v}}(0)+\frac{\rho_{\mathrm{w}} E}{\rho_{\mathrm{a}} H_{\text {moist }}} \Rightarrow \Delta q_{\mathrm{v}}, \\
& =-\frac{\rho_{\mathrm{w}}}{\rho_{\mathrm{a}} H_{\text {moist }}} \underbrace{\left(E_{\mathrm{wet}}-E_{\mathrm{dry}}\right)}_{\Delta E},
\end{aligned}
$$

where $H_{\text {moist }}$ is the vertical extent of the moistening process due to the accumulated surface evaporation $E$ and $q_{\mathrm{v}}(0)$ is the specific humidity at the initial time. By assuming that the moistening is confined to the PBL, so that $H_{\text {moist }}=H_{\text {front }}$ we can substitute Eq. (7) into Eq. (6) to obtain

$A_{\text {dry }}=\frac{\tau\left\langle u_{\text {front }}\right\rangle}{L_{\text {front }}} \Delta E$.

Our analysis thus indicates that the advection only depends on four terms: $\tau$, which is a constant; the difference in $E$ between the two patches, which can be estimated from Eq. (4) and which depends on the soil moisture; $L_{\text {front }} ;$ and $u_{\text {front }}$. In all simulations the front has a constant inland propagation of $L_{\text {front }} \simeq 100 \mathrm{~km}$, which corresponds to half of the patch size. More importantly, the front speed does not vary much with different surface heterogeneity gradients, against our initial expectations that motivated this study (see Introduction). For example, between the DA_20_100 and the DA_60_100 cases only a $3 \%$ relative decrease in the front speed is observed (not shown).

This counterintuitive behavior is related to the fact that cold pools lead to a noticeable acceleration of the front, as seen in Sect. 3.1. Although the front is initially triggered by the surface heterogeneity, and different surface heterogeneities may lead to different initial propagation velocities, the much faster cold pools end up determining the front velocity, thus masking the effect of the surface heterogeneity. This stands in agreement with what was found by Rieck et al. (2015), and in particular with the thermodynamic contribution of cold pools to the propagation speed of the front (their Eq. 1). Moreover, cold pools are distributed along the front and continuously fed by precipitation events, similarly to what happens in squall lines. Given this spatial organization, their strength and propagation do not depend on the surface state, as in the case for isolated convection (Gentine et al., 2016). Instead they solely depend on the state of the mid- to upper-troposphere (Peters and Hohenegger, 2017), which is also insignificantly modified by surface fluxes over the course of one diurnal cycle.

We can thus finally express advection simply as

$A_{\text {dry }}\left(\phi_{\text {dry }}\right)=\mathcal{B} \Delta E\left(\phi_{\text {dry }}\right)$,

where $\mathcal{B}=\tau\left\langle u_{\text {front }}\right\rangle / L_{\text {front }}$ is a proportionality constant that does not depend on soil moisture. Using the parameters $\mathcal{A}$, $\phi_{\mathrm{wp}}$ and $\phi_{\text {crit }}$ obtained from the fit of Eq. (4) (see Fig. 8) we can compute the difference $\Delta E\left(\phi_{\text {dry }}\right)$. Together with the values of $A_{\text {dry }}$ obtained in the simulations, the values of 
$\Delta E\left(\phi_{\text {dry }}\right)$ can be used to fit Eq. (9) and compute a value for the parameter $\mathcal{B}$ : in the $D A$ cases $\mathcal{B}=1.47$. This is smaller than the value that would be obtained by estimating instead $\left\langle u_{\text {front }}\right\rangle$ and $L_{\text {front }}$ directly, as this latter approximation does not take into account moisture losses due to advection.

Figure 9 shows the values of $A_{\text {dry }}$ and the fit performed using Eq. (9) for the basic set of experiments and for further cases, the latter used to test the finding that $\mathcal{B}$ does not depend on $\phi$ but solely on the atmospheric state. Overall the fit matches the variation in $A_{\text {dry }}$ with $\phi_{\text {dry }}$ remarkably well given the various assumptions. Both the simulated decrease in $A_{\text {dry }}$ with higher values of soil moisture and the flattening of advection by soil moisture lower than the wilting point are reproduced, although both effects seem to be overestimated by Eq. (9).

In the simulations where the initial value of $\phi_{\text {wet }}$ is reduced to just $70 \%$ of saturation the estimated value of $\mathcal{B}$ is almost the same as the one of the default configuration, confirming that $\mathcal{B}$ does not depend on soil moisture. Instead, in the ID_ cases (Table 3), which use a different atmospheric profile and hence support distinct cold pool strength, the value of $\mathcal{B}$ is reduced by about half.

\subsection{Computing the derivative of precipitation}

Equations (2), (4) and (9) can be combined in order to compute $P_{\text {dry }}$. We, however, are interested in its variation with soil moisture, $\frac{\partial P_{\text {dry }}}{\partial \phi_{\text {dry }}}$, which can be computed as

$$
\begin{aligned}
\frac{\partial P_{\mathrm{dry}}}{\partial \phi_{\mathrm{dry}}} & =\eta_{\mathrm{A}} \frac{\partial A_{\mathrm{dry}}}{\partial \phi_{\mathrm{dry}}}+\eta_{\mathrm{E}} \frac{\partial E_{\mathrm{dry}}}{\partial \phi_{\mathrm{dry}}}, \\
& =\eta_{\mathrm{A}} \frac{\partial}{\partial \phi_{\text {dry }}}\left(\mathcal{B}\left(E_{\mathrm{wet}}-E_{\mathrm{dry}}\right)\right)+\eta_{\mathrm{E}} \frac{\partial E_{\mathrm{dry}}}{\partial \phi_{\mathrm{dry}}}, \\
& =-\eta_{\mathrm{A}} \mathcal{B} \frac{\partial E_{\mathrm{dry}}}{\partial \phi_{\text {dry }}}+\eta_{\mathrm{E}} \frac{\partial E_{\mathrm{dry}}}{\partial \phi_{\text {dry }}} .
\end{aligned}
$$

Note that the derivation of Eq. (10) retains only one term of the difference given that $E_{\text {wet }}$ does not depend on $\phi_{\text {dry }}$. Using Eq. (4) it is straightforward to compute the derivative of $E_{\text {dry }}$ as

$$
\frac{\partial E_{\mathrm{dry}}}{\partial \phi_{\mathrm{dry}}}=\tau \mathcal{A}\left\langle Q_{\mathrm{net}}\right\rangle \times \begin{cases}0 & \text { for } \phi_{\mathrm{dry}}<\phi_{\mathrm{wp}} \\ \left(\phi_{\text {crit }}-\phi_{\mathrm{wp}}\right)^{-1} & \text { for } \phi_{\mathrm{wp}} \leq \phi_{\mathrm{dry}} \leq \phi_{\text {crit }} \\ 0 & \text { for } \phi_{\text {dry }}>\phi_{\text {crit }}\end{cases}
$$

which is a step-wise function consisting of constant values.

Equations (10) and (11) indicate that for $\phi_{\mathrm{dry}}<\phi_{\mathrm{wp}}$ and $\phi_{\text {dry }}>\phi_{\text {crit }}$ there is no change in precipitation with soil moisture independently of the value of the efficiencies. In contrast for $\phi_{\mathrm{wp}} \leq \phi_{\text {dry }} \leq \phi_{\text {crit }}$, the ratio $\frac{\partial E_{\text {dry }}}{\partial \phi_{\text {dry }}} \neq 0$ but still the derivative of precipitation with respect to soil moisture does not depend upon the soil moisture content itself. These findings contrast with the ones of Lintner et al. (2013), who found a minimum of the derivative for intermediate values of soil

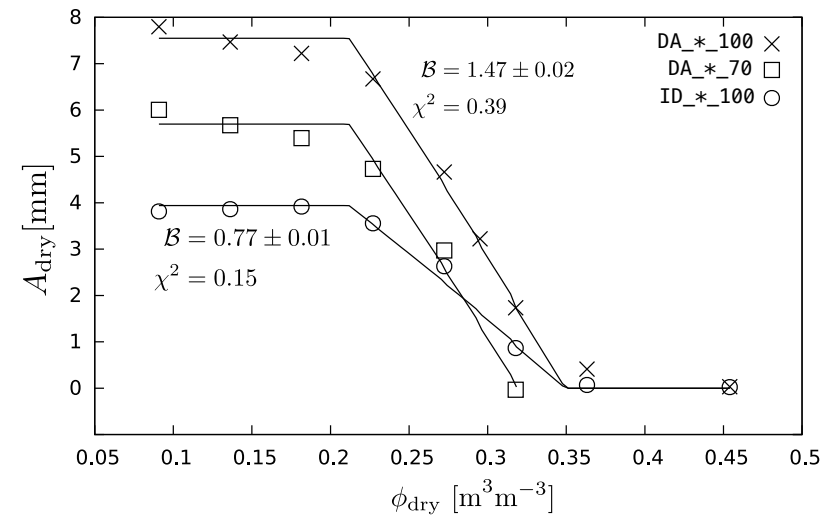

Figure 9. Fit of the advection in cases DA_*_100, DA_*_70 and ID_*_100. Symbols indicate the values obtained from the simulations while lines represent the fit performed using Eq. (9). The obtained values of $\mathcal{B}$ are reported in the insets, together with the absolute error and the $\chi^{2}$ value (see Fig. 8 for the definition). Note that for the DA_*_100 and DA_*_70 cases the fits yielded similar results; for this reason the obtained value for $\mathcal{B}$ is reported only once.

moisture. This is a consequence of the formulation of $E_{\mathrm{dry}}$ as a linear function of $\phi_{\text {dry }}$ and the fact that $A_{\text {dry }}$ also turned out to be a linear function of $\phi_{\text {dry }}$ as $u_{\text {front }}$ is constant. This remains true as long as the convection is strongly organized by the front associated with the mesoscale circulation and produces strong cold pools that end up determining the propagation velocity. It should be noted that, although the derivative $\frac{\partial P_{\text {dry }}}{\partial \phi_{\text {dry }}}$ does not depend on soil moisture, the value of precipitation $P_{\text {dry }}$ does indeed depend on soil moisture, as we will show later.

Coming back to Eqs. (10) and (11), we can now determine under which conditions $P_{\text {dry }}$ will increase or decrease.

$$
\frac{\partial P_{\text {dry }}}{\partial \phi_{\text {dry }}} \lessgtr 0 \Leftrightarrow-\eta_{\mathrm{A}} \mathcal{B}+\eta_{\mathrm{E}} \lessgtr 0 \Leftrightarrow \eta_{\mathrm{E}} \lessgtr \eta_{\mathrm{A}} \mathcal{B}
$$

The atmospheric conditions, through the terms $\eta_{\mathrm{A}}, \eta_{\mathrm{E}}$ and $\mathcal{B}$, determine whether increasing or decreasing the soil moisture of the dry patch is needed to increase the precipitation amount. Inserting the values of the efficiencies and of $\mathcal{B}$ obtained from the DA_ simulations in Eq. (12) confirms that $\frac{\partial P_{\text {dry }}}{\partial \phi_{\text {dry }}}<0$, which agrees with the simulated increase in precipitation with decreasing values of soil moisture. These results are generalized with the help of Fig. 10 for three different values of $\mathcal{B}$.

In Fig. 10 positive values indicate an increase in precipitation over the dry patch with soil moisture, and vice versa. Not surprisingly (see Eq. 12) using a value of $\mathcal{B}=1$ gives a symmetric picture where an increase in precipitation with soil moisture is obtained for those cases when $\eta_{\mathrm{E}}>\eta_{\mathrm{A}}$. This relationship is modified by the value of $\mathcal{B}$. 

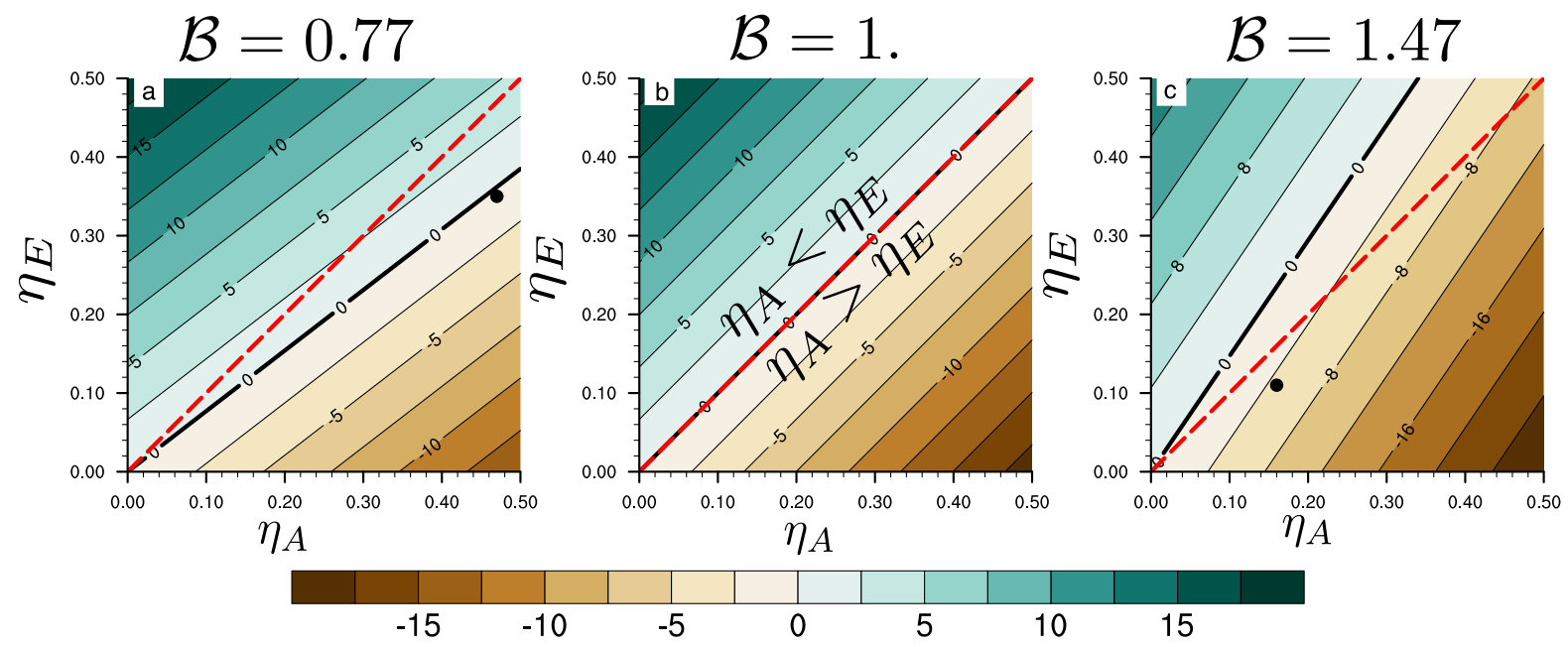

15

Figure 10. Contour plot of $\frac{\partial P_{\mathrm{dry}}}{\partial \phi_{\mathrm{dry}}}\left(\mathrm{mm} \mathrm{m}^{3} \mathrm{~m}^{-3}\right)$ as a function of $\eta_{\mathrm{A}}$ and $\eta_{\mathrm{E}}$ for different values of the parameter $\mathcal{B}$. The black points in $(\mathbf{a})$ and (c) are placed using the efficiencies obtained in the ID_ and DA_ cases, respectively. The dashed red line distinguishes the areas where $\eta_{\mathrm{A}}>\eta_{\mathrm{E}}$ and vice versa. Note the symmetric color scale and the thicker zero contour line.

Figure 10 overall shows that, as long as $\eta_{\mathrm{A}}>\eta_{\mathrm{E}}$, it is very unlikely to get a positive derivative. Only with values of $\mathcal{B}$ small enough, which would mean weaker and slower cold pools, the derivative may change sign even with $\eta_{\mathrm{A}}>\eta_{\mathrm{E}}$. This situation almost happens in the ID simulation, where the theory predicts a derivative close to zero. This agrees with the weaker sensitivity of precipitation to soil moisture observed in that case. Alternatively, to get a positive derivative, evaporation should become much more efficient than advection, i.e., $\eta_{\mathrm{E}} \gg \eta_{\mathrm{A}}$. This, however, did not happen in the performed simulations.

These findings already answer the main question posed in the Introduction and can be further generalized to the case when both $\phi_{\text {wet }}$ and $\phi_{\text {dry }}$ are changed at the same time. This allows one to investigate the dependency of precipitation on the soil moisture values of the two patches when the $\eta_{\mathrm{A}}$, $\eta_{\mathrm{E}}$ and $\mathcal{B}$ parameters are fixed. First of all, $\frac{\partial P_{\text {dry }}}{\partial \phi_{\text {wet }}}$ can be computed with the same method as before:

$\frac{\partial P_{\text {dry }}}{\partial \phi_{\text {wet }}}=\eta_{\mathrm{A}} \mathcal{B} \frac{\partial E_{\text {wet }}}{\partial \phi_{\text {wet }}}$

given that the evaporation over the dry patch does not depend on the soil moisture of the wet patch. Second, the two derivatives $\frac{\partial P_{\mathrm{dry}}}{\partial \phi_{\mathrm{wet}}}$ and $\frac{\partial P_{\mathrm{dry}}}{\partial \phi_{\mathrm{dry}}}$ can be combined to obtain the total precipitation change over the dry patch.

$$
\begin{aligned}
\Delta P_{\text {dry }} & =\frac{\partial P_{\text {dry }}}{\partial \phi_{\text {dry }}} \Delta \phi_{\text {dry }}+\frac{\partial P_{\text {dry }}}{\partial \phi_{\text {wet }}} \Delta \phi_{\text {wet }}, \\
& =\left(\eta_{\mathrm{E}}-\eta_{\mathrm{A}} \mathcal{B}\right) \frac{\partial E_{\text {dry }}}{\partial \phi_{\text {dry }}} \Delta \phi_{\text {dry }}+\eta_{\mathrm{A}} \mathcal{B} \frac{\partial E_{\text {wet }}}{\partial \phi_{\text {wet }}} \Delta \phi_{\text {wet }} .
\end{aligned}
$$

Assuming that the soil type of both patches is the same, only the case $\phi_{\mathrm{wp}}<\phi_{\text {dry, wet }}<\phi_{\text {crit }}$ is of interest. The other cases either revert to the previously discussed case (Eq. 12) or reduce to the trivial solution where only $\Delta \phi_{\text {wet }}$ is affecting $\Delta P_{\text {dry }}$. For $\phi_{\mathrm{wp}}<\phi_{\text {dry }}$ wet $<\phi_{\text {crit }}$ we obtain $\frac{\partial E_{\text {dry }}}{\partial \phi_{\text {dry }}}=\frac{\partial E_{\text {wet }}}{\partial \phi_{\text {wet }}}$. Thus, changes in precipitation in our idealized model can be formulated as

$\Delta P_{\text {dry }}=\tau \frac{\mathcal{A}\left\langle Q_{\text {net }}\right\rangle}{\phi_{\text {crit }}-\phi_{\mathrm{wp}}}\left(\left(\eta_{\mathrm{E}}-\eta_{\mathrm{A}} \mathcal{B}\right) \Delta \phi_{\text {dry }}+\eta_{\mathrm{A}} \mathcal{B} \Delta \phi_{\mathrm{wet}}\right)$.

The behavior of Eq. (15) as a function of $\Delta \phi_{\text {dry }}, \Delta \phi_{\text {wet }}$ and $\mathcal{B}$ is investigated with the help of Fig. 11. In the default configuration described in Sect. 3.1 the soil moisture of the wet patch was kept constant, i.e., $\Delta \phi_{\text {wet }}=0$, while the soil moisture of the dry patch was increased, i.e., $\Delta \phi_{\text {dry }}>0$. Figure 11a shows that, in the aforementioned case, $\Delta P_{\text {dry }}$ is negative, as in our simulations. In this case decreasing $\phi_{\text {dry }}$ and increasing $\phi_{\text {wet }}$ is the most efficient way to increase precipitation.

Figure $11 \mathrm{~b}$ presents the case characteristic of the simulations performed with the ID sounding. The flattening of the contour lines shows that there is little sensitivity to $\phi_{\mathrm{dry}}$, as previously discussed. Mainly increasing $\phi_{\text {wet }}$ would allow precipitation to increase. In the extreme case where $\mathcal{B}$ is further reduced (Fig. 11c) the picture partly reverses. Both soil moisture of the wet and of the dry patch should be increased to sustain an increase in precipitation, as evaporation becomes now relevant and advection has a negligible contribution.

Figure 11 thus indicates that, in any case, the soil moisture of the wet patch should be increased to get more precipitation on the dry patch. The response to changes in soil moisture of the dry patch is more subtle, and the combination of the two responses can lead to positive or negative coupling depending on the atmosphere state. This may explain why in reality 


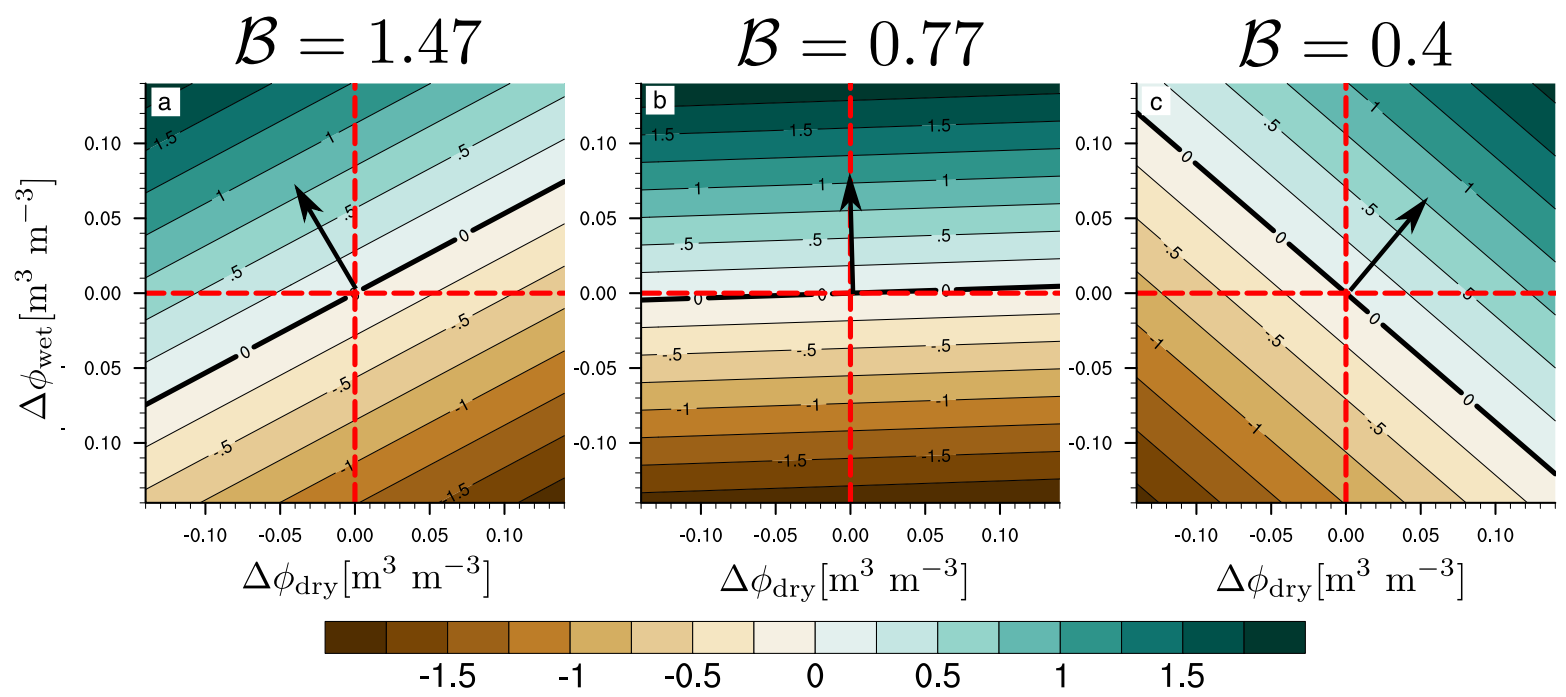

Figure 11. $\Delta P_{\text {dry }}$ as a function of $\Delta \phi_{\text {dry }}$ and $\Delta \phi_{\text {wet }}$ for different values of the parameter $\mathcal{B}$. The $x$ and $y$ axes represent the variation in $\phi_{\text {dry }}$ and $\phi_{\mathrm{wet}}$, respectively. Note that the maximum variation is $\phi_{\text {crit }}-\phi_{\mathrm{wp}}$, as $\Delta P_{\mathrm{dry}}$ is computed for the regime $\phi_{\mathrm{wp}}<\phi_{\mathrm{dry} \text {, wet }}<\phi_{\text {crit }}$. The red dashed lines indicate no variation in the soil moisture of either one of the patches. The efficiencies are set to $\left(\eta_{\mathrm{A}}, \eta_{\mathrm{E}}\right)=(0.16,0.11)$ in $(\mathbf{a})$ and to $\left(\eta_{\mathrm{A}}, \eta_{\mathrm{E}}\right)=(0.47,0.35)$ in $(\mathbf{b})$ and (c) to match the simulation results. The black arrows indicate the direction of maximum growth, i.e., when an increase in precipitation is expected.

both signs of the coupling are observed with different atmospheric states.

\section{Conclusions}

Motivated by the ambiguous relationship between soil moisture, soil moisture heterogeneity and precipitation we designed idealized simulations of a convective diurnal cycle that make use of a coupled configuration of an atmospheric LES (large eddy simulation) model and a land-surface model. The heterogeneity in the land surface was prescribed by dividing the domain into two patches with different initial values of soil moisture. Inspired by the results of the simulations, we specifically wanted to derive a simple conceptual model that retains the minimum parameters that control precipitation over a spatially drier patch. Moreover, we wanted to use this model to understand which is the most efficient way to increase precipitation by acting on soil moisture given the opposite control of soil moisture on advection and evaporation.

Since the main potential sources contributing to precipitation are consisted of remote moisture advection by the mesoscale circulation triggered by the soil moisture heterogeneity and local evaporation, we first aim at disentangling the effects of these two on precipitation. Results from the simulations show, as expected, that the moisture advection over the dry patch decreases with increasing local soil moisture, while evaporation increases. The interplay between these two effects produces a decrease in precipitation with increasing values of local soil moisture for the considered case.
More importantly the simulation results indicated that such a decrease can only be correctly reproduced by assuming that advection and evaporation processes contribute differently to precipitation. Hence we model precipitation as the sum of advection and evaporation each weighed by its own efficiency (see Eq. 2). By using two efficiencies, they become independent of soil moisture and only dependent on the initial atmospheric state.

As a second step we conceptualize the variations in evaporation and advection with soil moisture. Evaporation can be approximated using the bucket model owing to Budyko (1961) (see Eq. 3). The advection is estimated as the product of the breeze front velocity and the gradient in near-surface specific humidity (see Eq. 5). A priori we would have expected a squared dependency of advection on soil moisture since both the velocity of the front and the gradient in specific humidity should be related to soil moisture. However, it turns out that the velocity of the front is independent of soil moisture as the development of convection at the breeze front and the generation of strong cold pools lead to a strong acceleration of the front that fully masks the effect of the initial surface heterogeneity.

Putting all the results together indicates that the derivative of precipitation with respect to the soil moisture of the dry patch does not depend on the actual soil moisture value. This is due to the fact that the functional forms of advection and evaporation end up being linear functions of soil moisture. The idealized model is valid as long as the evaporation keeps its linearity as a function of soil moisture and the propagation 
speed of the front does not depend on the surface heterogeneity gradient, meaning strong enough cold pools.

The parameters that control the variations in precipitation with local soil moisture are the aforementioned efficiencies and a scale parameter that defines the magnitude of the advection. All these parameters depend solely on the atmospheric state. According to the values of these parameters, as estimated from the simulations, the most efficient way to increase precipitation over the dry patch is to decrease the soil moisture of the dry patch. Thus, one can say that, in order to have more precipitation over spatially drier areas, more precipitation should first fall on spatially wetter ones. In other words, the most efficient way to obtain more precipitation over dry areas is to let them dry out for a long time so that a stronger gradient can build up and thus produce more explosive convective events due to a stronger mesoscale circulation.
However, if either the efficiency of evaporation becomes much larger than the one of advection or the scale parameter that defines the importance of advection decreases under a certain threshold then the response of precipitation can be reversed. Although we did not find any evidence of this behavior for the two atmospheric profiles tested in this work it would be interesting as a next step to derive the three parameters predicted by the conceptual model from more realistic simulations to infer the frequency of occurrence of the various precipitation regimes.

Data availability. Primary data and scripts used in the analysis are archived by the Max Planck Institute for Meteorology and can be obtained by contacting publications@mpimet.mpg.de. 


\section{Appendix A: Computation of the advection as residual term}

The advection of every tracer is computed directly from the moisture balance equation as a residual. We use the following formulation, which applies for a certain point $(x, y)$ over a 2 dimensional domain:

$$
\begin{aligned}
& \frac{1}{\rho_{\mathrm{w}}} \int_{0}^{\tau} \int_{0}^{H} \frac{\mathrm{D} q_{\mathrm{tot}}}{\mathrm{D} t} \rho_{\mathrm{a}} \mathrm{d} z \mathrm{~d} t=\frac{1}{\rho_{\mathrm{w}}} \int_{0}^{\tau} \int_{0}^{H}\left[\frac{\partial q_{\mathrm{tot}}}{\partial t}+\boldsymbol{v} \cdot \nabla q_{\mathrm{tot}}\right] \\
& \rho_{\mathrm{a}} \mathrm{d} z \mathrm{~d} t=E-P,
\end{aligned}
$$

where D indicates the total derivative, $P(\mathrm{~m})$ is the accumulated precipitation, $E(\mathrm{~m})$ the accumulated evaporation, $q_{\text {tot }}\left(\mathrm{kg} \mathrm{kg}^{-1}\right)$ represents the sum of all tracers (water vapor $q_{\mathrm{v}}$, clouds $q_{\mathrm{c}}$, rain $q_{\mathrm{r}}$, snow $q_{\mathrm{s}}$, ice $q_{\mathrm{i}}$, graupel $q_{\mathrm{g}}$ and hail $\left.q_{\mathrm{h}}\right)$ mixing ratios, $\rho_{\mathrm{w}}\left(\mathrm{kg} \mathrm{m}^{-3}\right)$ the density of water, $\rho_{\mathrm{a}}\left(\mathrm{kg} \mathrm{m}^{-3}\right)$ the air density and $\boldsymbol{v}$ the velocity of air as a vector. $H$ indicates the top of the simulation domain and $\tau$ the length of the accumulation period ( $18 \mathrm{~h}$ in our experiments). The total derivative can be divided into its advective term:

$A \equiv-\frac{1}{\rho_{\mathrm{w}}} \int_{0}^{\tau} \int_{0}^{H} \boldsymbol{v} \cdot \nabla q_{\mathrm{tot}} \rho_{\mathrm{a}} \mathrm{d} z \mathrm{~d} t$

and the local derivative:

$$
\frac{1}{\rho_{\mathrm{w}}} \int_{0}^{\tau} \int_{0}^{H} \frac{\partial q_{\mathrm{tot}}}{\partial t} \rho_{\mathrm{a}} \mathrm{d} z \mathrm{~d} t=A+E-P .
$$

In both Eqs. (A2) and (A3) the variables $A, E$ and $P$ are solely functions of $x$ and $y$, whereas $q_{\text {tot }}$ depends also on time $t$ and on the vertical coordinate $z$. We can eliminate the dependency on $x$ and $y$ by applying an average operator over a certain area, indicated with the subscript ${ }_{\text {area }}$ :

$$
\left.\frac{1}{\rho_{\mathrm{W}}} \int_{0}^{\tau} \int_{0}^{H} \frac{\partial q_{\text {tot }}}{\partial t}\right|_{\text {area }} \rho_{\mathrm{a}} \mathrm{d} z \mathrm{~d} t .=A_{\text {area }}+E_{\text {area }}-P_{\text {area }} .
$$

In the main text we use as area either the full domain, denoted with the subscript dom, or the dry patch only, denoted by the subscript dry. By indicating the weighted vertical integral of $q_{\text {tot }}$ as $\bar{q}_{\text {tot }} \equiv \int_{0}^{H} q_{\text {tot }} \rho_{\mathrm{a}} \mathrm{d} z$ we can further simplify the previous equation to

$\left.\frac{1}{\rho_{\mathrm{w}}} \int_{0}^{\tau} \frac{\partial \bar{q}_{\text {tot }}}{\partial t}\right|_{\text {area }} \mathrm{d} t=A_{\text {area }}+E_{\text {area }}-P_{\text {area }}$.

Although other studies only considered the advection of water vapor, i.e., of $\bar{q}_{\mathrm{v}}$, in order to close the balance it is necessary to consider all species. In fact, although $\bar{q}_{\mathrm{i}}, \bar{q}_{\mathrm{g}}, \bar{q}_{\mathrm{h}}$, and $\bar{q}_{\mathrm{s}}$ are orders of magnitude smaller than $\bar{q}_{\mathrm{v}}, \bar{q}_{\mathrm{c}}$, and $\bar{q}_{\mathrm{r}}$, their variations over time are not, so that neglecting these terms in Eq. (A5) would lead to an imbalance.

From the $5 \mathrm{~min}$ simulation output we use Eq. (A5) and estimate the advection as the residual $R_{\text {area }}=\left.\frac{1}{\rho_{\mathrm{w}}} \int_{0}^{\tau} \frac{\partial \bar{q}_{\text {tot }}}{\partial t}\right|_{\text {area }} \mathrm{d} t+P_{\text {area }}-E_{\text {area }} \equiv A_{\text {area }}$. We verify that, when averaged over the entire domain, $R_{\mathrm{dom}}=0$. 
Competing interests. The authors declare that they have no conflict of interest.

Acknowledgements. This research was supported by the HErZ (Hans-Ertel-Zentrum for Weather Research), a collaborative project involving universities across Germany, the DWD (Deutscher Wetterdienst) and funded by the BMVI (Federal Ministry of Transport and Digital Infrastructure). The simulations were performed using the facilities of the DKRZ (Deutsches Klimarechenzentrum) and in particular the new supercomputer Mistral. We thank two anonymous reviewers for their comments and corrections.

The article processing charges for this open-access publication were covered by the Max Planck Society.

Edited by: Carlo De Michele

Reviewed by: two anonymous referees

\section{References}

Avissar, R. and Liu, Y.: Three-dimensional numerical study of shallow convective clouds and precipitation induced by land surface forcing, J. Geophys. Res.-Atmos., 101, 7499-7518, 1996.

Budyko, M. I.: The heat balance of the earth's surface, Soviet Geography, 2, 3-13, 1961.

Budyko, M. I.: Climate and Life, Academic Press, New York, 1974.

Chen, F. and Avissar, R.: Impact of land-surface moisture variability on local shallow convective cumulus and precipitation in largescale models, J. Appl. Meteorol., 33, 1382-1401, 1994.

Cioni, G. and Hohenegger, C.: Effect of soil moisture on diurnal convection and precipitation in Large-Eddy Simulations, J. Hydrometeorol., 18, 1885-1903, https://doi.org/10.1175/JHMD-16-0241.1, 2017.

Crosman, E. T. and Horel, J. D.: Sea and lake breezes: a review of numerical studies, Bound.-Lay. Meteorol., 137, 1-29, 2010.

Doms, G., Forstner, J., Heise, E., Herzog, H.-J., Mironov, D., Raschendorfer, M., Reinhardt, T., Ritter, B., Schrodin, R., Schulz, J.-P., and Vogel, G.: A Description of the Nonhydrostatic Regional COSMO Model. Part II: Physical Parametrization, Consortium for Small-Scale Modeling, http://www.cosmo-model.org/content/model/documentation/ core/cosmoPhysParamtr.pdf (last access: June 2018), 2011.

Findell, K. L. and Eltahir, E. A.: Atmospheric controls on soil moisture-boundary layer interactions. Part I: Framework development, J. Hydrometeorol., 4, 552-569, 2003.

Froidevaux, P., Schlemmer, L., Schmidli, J., Langhans, W., and Schär, C.: Influence of the background wind on the local soil moisture-precipitation feedback, J. Atmos. Sci., 71, 782-799, 2014.

Gentine, P., Garelli, A., Park, S.-B., Nie, J., Torri, G., and Kuang, Z.: Role of surface heat fluxes underneath cold pools, Geophys. Res. Lett., 43, 874-883, 2016.

Guillod, B. P., Orlowsky, B., Miralles, D. G., Teuling, A. J., and Seneviratne, S. I.: Reconciling spatial and temporal soil moisture effects on afternoon rainfall, Nat. Commun., 6, 6443, https://doi.org/10.1038/ncomms7443, 2015.
Heinze, R., Dipankar, A., Henken, C. C., Moseley, C., Sourdeval, O., Trömel, S., Xie, X., Adamidis, P., Ament, F., Baars, H., Barthlott, C., Behrendt, A., Blahak, U., Bley, S., Brdar, S., Brueck, M., Crewell, S., Deneke, H., Di Girolamo, P., Evaristo, R., Fischer, J., Frank, C., Friederichs, P., Göcke, T., Gorges, K., Hande, L., Hanke, M., Hansen, A., Hege, H., Hoose, C., Jahns, T., Kalthoff, N., Klocke, D., Kneifel, S., Knippertz, P., Kuhn, A., van Laar, T., Macke, A., Maurer, V., Mayer, B., Meyer, C. I., Muppa, S. K., Neggers, R. A., Orlandi, E., Pantillon, F., Pospichal, B., Röber, N., Scheck, L., Seifert, A., Seifert, P., Senf, F., Siligam, P., Simmer, C., Steinke, S., Stevens, B., Wapler, K., Weniger, M., Wulfmeyer, V., Zängl, G., Zhang, D., and Quaas, J.: Large-eddy simulations over Germany using ICON: A comprehensive evaluation, Q. J. Roy. Meteorol. Soc., 143, 69-100, https://doi.org/10.1002/qj.2947, 2017.

Koster, R. D., Dirmeyer, P. A., Guo, Z., Bonan, G., Chan, E., Cox, P., Gordon, C., Kanae, S., Kowalczyk, E., Lawrence, D., Liu, P., Lu, S., Malyshev, S., McAvaney, B., Mitchell, K., Mocko, D., Oki, T., Oleson, K., Pitman, A., and Yamada, T.: Regions of strong coupling between soil moisture and precipitation, Science, 305, 1138-1140, 2004.

Lintner, B. R., Gentine, P., Findell, K. L., D’Andrea, F., Sobel, A. H., and Salvucci, G. D.: An idealized prototype for large-scale land-atmosphere coupling, J. Climate, 26, 2379-2389, 2013.

Lynn, B. H., Tao, W.-K., and Wetzel, P. J.: A study of landscapegenerated deep moist convection, Mon. Weather Rev., 126, 928 942, 1998.

Manabe, S.: Climate and the ocean circulation 1: i. The atmospheric circulation and the hydrology of the earth's surface, Mon Weather Rev., 97, 739-774, 1969.

Neelin, J. D. and Zeng, N.: A quasi-equilibrium tropical circulation model - Formulation, J. Atmos. Sci., 57, 1741-1766, 2000.

Peters, K. and Hohenegger, C.: On the Dependence of Squall-Line Characteristics on Surface Conditions, J. Atmos. Sci., 74, 22112228, 2017.

Rieck, M., Hohenegger, C., and Gentine, P.: The effect of moist convection on thermally induced mesoscale circulations, Q. J. Roy. Meteorol. Soc., 141, 2418-2428, 2015.

Robinson, F. J., Sherwood, S., and Li, Y.: Resonant response of deep convection to surface hot spots, J. Atmos. Sci., 65, 276286, 2008.

Robinson, F. J., Patterson, M. D., and Sherwood, S.: A numerical modeling study of the propagation of idealized sea-breeze density currents, J. Atmos. Sci., 70, 653-668, 2013.

Schär, C., Lüthi, D., Beyerle, U., and Heise, E.: The soilprecipitation feedback: A process study with a regional climate model, J. Climate, 12, 722-741, 1999.

Schlemmer, L., Hohenegger, C., Schmidli, J., and Schär, C.: Diurnal equilibrium convection and land surface-atmosphere interactions in an idealized cloud-resolving model, Q. J. Roy. Meteorol. Soc., 138, 1526-1539, 2012.

Schulz, J.-P., Vogel, G., Becker, C., Kothe, S., Rummel, U., and Ahrens, B.: Evaluation of the ground heat flux simulated by a multi-layer land surface scheme using high-quality observations at grass land and bare soil, Meteorol. Z., 25, 607-620, https://doi.org/10.1127/metz/2016/0537, 2016.

Segal, M. and Arritt, R.: Nonclassical mesoscale circulations caused by surface sensible heat-flux gradients, B. Am. Meteorol. Soc., 73, 1593-1604, 1992. 
Seneviratne, S. I., Corti, T., Davin, E. L., Hirschi, M., Jaeger, E. B., Lehner, I., Orlowsky, B., and Teuling, A. J.: Investigating soil moisture-climate interactions in a changing climate: A review, Earth-Sci. Rev., 99, 125-161, 2010.

Taylor, C. M., de Jeu, R. A., Guichard, F., Harris, P. P., and Dorigo, W. A.: Afternoon rain more likely over drier soils, Nature, 489, 423-426, 2012.

Tuttle, S. and Salvucci, G.: Confounding factors in determining causal soil moisture-precipitation feedback, Water Res. Res., 53, 5531-5544, https://doi.org/10.1002/2016WR019869, 2017.
Wei, J., Su, H., and Yang, Z.-L.: Impact of moisture flux convergence and soil moisture on precipitation: a case study for the southern United States with implications for the globe, Clim. Dynam., 46, 467-481, 2016.

Yan, H. and Anthes, R. A.: The effect of variations in surface moisture on mesoscale circulation, Mon. Weather Rev., 116, 192-208, 1988.

Zängl, G., Reinert, D., Rípodas, P., and Baldauf, M.: The ICON (ICOsahedral Non-hydrostatic) modelling framework of DWD and MPI-M: Description of the non-hydrostatic dynamical core, Q. J. Roy. Meteorol. Soc., 141, 563-579, 2015. 